\title{
La C2I ou rendre l'étalonnage conditionnel
}

\section{IIC, a monitoring method for conditional calibrations}

\author{
Pascal COQUET
}

ACEI, 6 bis rue Jean Zay, 64000 Pau, France

LA Métrologie, 36 rue des frères Wright, 64000 Pau, France

\begin{abstract}
Inspirée des comparaisons inter-laboratoires, la comparaison inter-instruments (C2I) est une méthode de surveillance d'équipements de mesure au sens large. Détection de dérive ou de dégradation de caractéristiques métrologiques, optimisation de périodicités d'étalonnage... Plus puissante qu'une carte de contrôle individuelle, la méthode traite un lot d'instruments de même famille. L'article présente la méthode telle qu'elle a été pensée et développée par le groupe de travail de la commission de métrologie de l'AFNOR (fascicule FD X07-41).
\end{abstract}

$\underline{\text { Note }}$ : le terme " instrument 》 utilisé en C2I est à prendre au sens large, la méthode s'appliquant à tous types d'équipements de mesure.

\section{Principe}

Basée sur le principe que des instruments de mesure de même famille n'ont statistiquement aucune raison de dériver dans le même sens et de la même valeur durant le même intervalle de temps, la C2I emprunte à l'ISO 5725 ses tests de valeurs aberrantes pour détecter d'éventuels instruments « douteux ».

\section{Applications}

Son application la plus évidente est la surveillance de dérive d'instruments entre deux confirmations métrologiques; mais son potentiel est nettement plus élevé : selon la manière dont elle est mise en œuvre, elle peut répondre à certains cas de raccordements difficiles, lever un doute sur un instrument ou encore optimiser les périodicités d'étalonnage.

\subsection{Détection de dérive}

Comparer un lot d'instruments permet de détecter celui ou ceux qui sont statistiquement différents. Si ces instruments n'ont pas été détectés lors de la précédente confirmation (étalonnage, C2I...), c'est qu'ils ont probablement dérivé.

\subsection{Levée de doute}

Si un instrument est mis en doute lors d'un mesurage, la C2I peut, en l'absence d'étalon, confirmer ou infirmer ce doute.

\subsection{Optimisation de la périodicité}

Le fait de réaliser des C2I sur une famille d'instruments permet d'optimiser leur périodicité d'étalonnage d'une manière inédite: supposons un lot d'instruments ayant été étalonnés au moins une fois (au minimum à la mise en service), tant que les résultats de C2I successives ne montrent pas de différence statistique entre eux, on peut légitimement penser que leur raccordement n'est pas remis en question et que, dans ces conditions, le réétalonnage n'est pas nécessaire, au moins jusqu'à la prochaine comparaison. La C2I devient alors un outil d'optimisation d'une périodicité devenue conditionnelle.

Note : Il faut évidemment, dans ce cas, être en mesure de prouver que tous les instruments ne risquent pas de dériver de la même quantité, dans le même sens. Ce cas est peu probable mais doit être considéré.

\subsection{Surveillance d'étalons}

Il est également possible d'optimiser la périodicité d'étalonnage d'étalons ou d'équipements dont l'incertitude d'utilisation est connue et utilisée. Dans ce cas, il convient, au terme de la comparaison, de calculer la variance inter-instruments et de s'assurer que sa racine carrée est inférieure à l'incertitude cible. Dans le cas contraire, il existe un risque que la $\mathrm{C} 2 \mathrm{I}$ ne soit pas à même de détecter un problème potentiel sur l'un des étalons.

\subsection{Etalonnage dans les cas difficiles}

Il existe de nombreux cas d'instruments installés sur des process industriels, dont la dépose s'avère difficile ou coûteuse; des cas où l'étalonnage sur site n'étant pas

(C) The Authors, published by EDP Sciences. This is an open access article distributed under the terms of the Creative Commons Attribution 
possible, il faut «sectionner» la chaine de mesure pour n'envoyer que le capteur en étalonnage (lecture sur système d'acquisition); des cas où le fait même de déposer un instrument modifie les paramètres du process (par exemple une sonde de régulation qui ne serait pas replacée exactement au même endroit).

Dans tous ces cas, la C2I peut apporter une solution adaptée.

Il est par exemple possible de comparer des thermomètres montés sur des lignes de process. Dans ce cas précis, l'objet à mesurer (milieu de comparaison) n'étant pas commun, un thermomètre supplémentaire, appelé intermédiaire, est nécessaire; la comparaison porte alors sur les écarts mesurés entre celui-ci et chacun des thermomètres en ligne.

\section{Sélection des participants}

La méthode repose sur une hypothèse forte : un lot d'instruments n'a statistiquement aucune raison de dériver dans le même sens et dans la même proportion durant le même intervalle de temps.

La sélection des instruments participant à la comparaison doit être réalisée avec le plus grand soin de manière à respecter cette hypothèse : on évitera par exemple de ne choisir que des instruments issus du même lot de fabrication car ils auraient précisément des chances de porter en eux les mêmes défauts, voire de dériver de manière quelque peu synchronisée. Cette étape est primordiale ; le non respect de l'hypothèse de départ peut mener à des décisions préjudiciables sur le plan du management de qualité.

Exemples de conditions à respecter :

- Instruments de même famille (mesurant la même grandeur)

- Instruments de caractéristiques métrologiques équivalentes $^{1}$ (résolutions, exactitude, etc... comparables).

- Instruments hétérogènes (marques et modèles différents) de manière à éviter le risque de dérive synchronisée

\section{Méthode}

Sachant que la comparaison doit faire ressortir uniquement les défauts intrinsèques aux instruments, il est impératif de définir une méthode maîtrisée, établie afin de garantir la stabilité de tous les facteurs d'influence.

\footnotetext{
${ }^{1}$ II faut garder à l'esprit que la C2I ne pourra mettre en évidence des défauts inférieurs aux performances du moins performant des instruments
}

Lorsque certains facteurs ne peuvent être maîtrisés, ils devront être déterminés et pris en compte dans les calculs, voir par exemple l'exemple des cas difficiles (cf. $\S 1.5)$ où l'on intègre un instrument supplémentaire, appelé intermédiaire, pour mesurer la température du produit qui n'est pas forcément identique en tous points du process. Il convient d'ailleurs de distinguer deux cas. Soit les températures mesurées sont sensiblement les mêmes et un instrument intermédiaire non raccordé conviendra, soit elles sont très différentes et il faudra alors s'assurer que l'erreur de justesse de l'instrument intermédiaire est suffisamment constante sur la plage de température considérée pour ne pas introduire de biais dans l'estimation des écarts moyens.

\section{Les tests}

Cette partie est illustrée par une C2I menée sur un lot de 7 pipettes automatiques de $2 \mathrm{ml}$. Chacune prélève un volume de $2 \mathrm{ml}$ d'eau dans un récipient puis le déverse dans un second récipient posé sur une balance à $0,1 \mathrm{mg}$ dont on ne se soucie pas du raccordement. Les conditions suivantes sont réalisées :

- Le laboratoire est régulé en température et hygrométrie

- L'eau a reposé suffisamment longtemps dans le premier récipient pour être en équilibre thermique

- Le second récipient est vidé après chaque série et la balance remise à zéro entre chaque mesure

- Les cônes sont saturés et les pipettes utilisées selon les BPL (bonnes pratiques de laboratoire).

La méthode retenue consiste en un test de Mandel qui se déroule en 2 étapes : un test des variances (statistique $\mathrm{k}$ ) et un test des moyennes (statistique h).

Le test de Mandel est un test graphique particulièrement pratique et simple à interpréter. Un autre avantage est que son test $\mathrm{k}$ recherche uniquement les variances trop élevées, la détection d'une variance anormalement faible n'ayant, dans le cadre d'une C2I, pas d'intérêt. En revanche, le test de Mandel impose une condition: que le nombre de mesures réalisées soit identique pour chaque instrument.

Résultats :

\begin{tabular}{|c|c|c|c|c|c|c|}
\hline M012 & M014 & M015 & M060 & M061 & M062 & M068 \\
\hline 2,0103 & 1,9676 & 1,9731 & 2,0087 & 2,0041 & 2,0199 & 1,9990 \\
2,0129 & 1,9689 & 1,9654 & 1,9981 & 2,0112 & 1,9958 & 2,0072 \\
2,0112 & 1,9667 & 1,9593 & 2,0089 & 2,0197 & 2,0049 & 2,0161 \\
1,9945 & 1,9985 & 1,9664 & 1,9961 & 2,0107 & 1,9957 & 2,0219 \\
2,0130 & 1,9588 & 1,9764 & 1,9824 & 2,0107 & 2,0008 & 1,9964 \\
2,0282 & 1,9606 & 1,9623 & 2,0031 & 2,0179 & 1,9912 & 2,0085 \\
2,0231 & 1,9827 & 1,9719 & 2,0172 & 1,9992 & 2,0045 & 2,0076 \\
\end{tabular}




\begin{tabular}{|l|l|l|l|l|l|l|}
2,0217 & 1,9627 & 2,0183 & 2,0003 & 2,0056 & 2,0090 & 2,0127 \\
2,0322 & 1,9833 & 2,0214 & 2,0071 & 2,0133 & 2,0066 & 2,0177 \\
2,0283 & 1,9626 & 1,9903 & 2,0075 & 2,0147 & 2,0106 & 2,0113 \\
\hline
\end{tabular}

Note: dans un souci de simplification et afin de se concentrer sur les propriétés de la C2I,

Note: la variation maximale de température de l'eau estimée au cours de la comparaison $\left(1^{\circ} \mathrm{C}\right)$ entraîne une variation potentielle de 0,0004 ml, valeur qui, même si elle était atteinte, serait noyée dans les écarts-types intrainstruments, donc négligeable. La température de l'eau est donc supposée constante.

Note: la température de l'eau n'étant pas mesurée, on travaille directement sur les valeurs de masses, images $d u$ volume à température constante.

Statistique k (variances) :

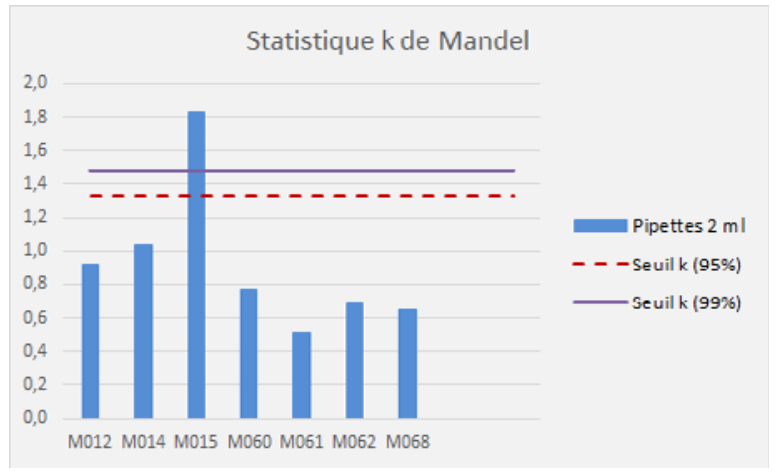

Statistique h (moyennes) :

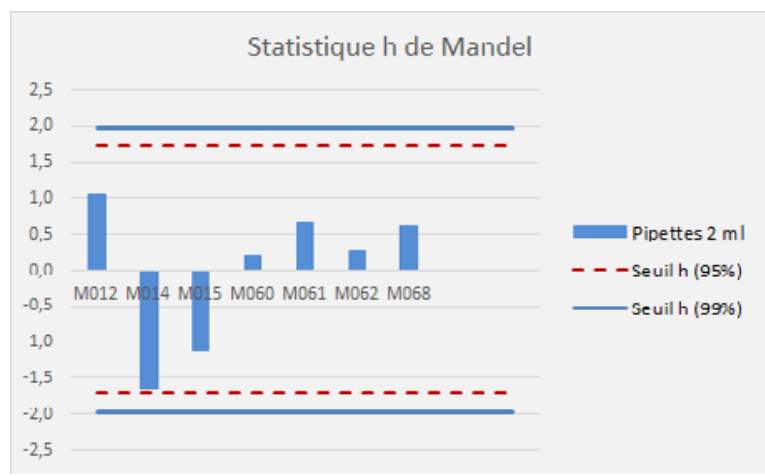

Le test $\mathrm{k}$ fait ressortir que la dispersion de la pipette M015 est anormalement élevée au regard des autres. Cette pipette nécessitant un examen approfondi, elle est écartée du test, lequel est poursuivi sans elle.
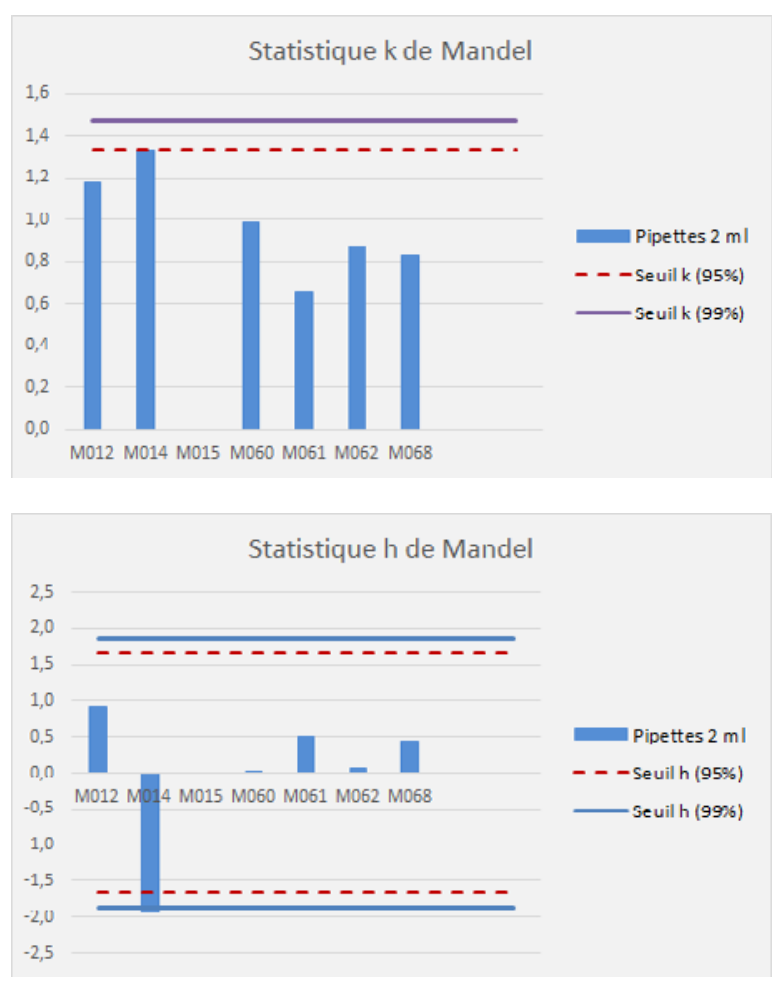

Le nouveau test $\mathrm{k}$ (sans M015) détecte la pipette M014 sur le seuil à $95 \%$, confirmé par le test $\mathrm{h}$ sur les moyennes (dépassement du seuil à 99\%).

La pipette M014 écartée à son tour, le test est poursuivi :

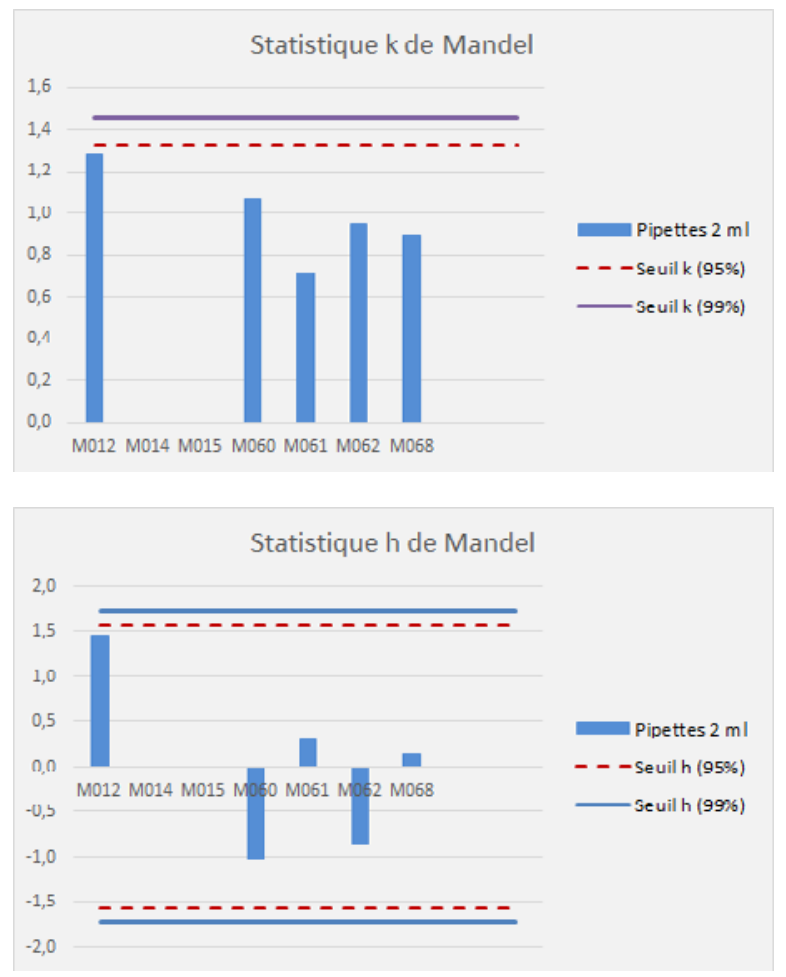

Les tests $\mathrm{k}$ et $\mathrm{h}$ valident le fait que les pipettes restantes ne sont pas statistiquement différentes. 


\section{Reproductibilité}

Selon le but de la C2I, Il peut être intéressant, afin de compléter le résultat du test, d'évaluer l'écart-type de reproductibilité de la famille étudiée.

Dans notre exemple, on calcule un écart-type moyen de répétabilité $\mathrm{sr}=0,0123 \mathrm{ml}$ et un écart-type de reproductibilité de $0,0166 \mathrm{ml}$.

Note: la moyenne générale des mesures réalisées (1,9995 ml) n'a de sens que si la balance utilisée est raccordée.

\section{Le résultat}

Deux constats s'imposent à l'issue de la comparaison :

- Les pipettes M014 et M015 sont détectées comme différentes des autres

- Les 5 pipettes restantes ne sont statistiquement pas différentes entre elles

Que signifie ce résultat? Que faire des pipettes détectées ? Comment interpréter la « non différence » des pipettes restantes?

\section{L’interprétation}

Tout dépend du but recherché !

Quelques exemples :

Dans le cas d'une simple comparaison, d'une étude de l'homogénéité d'une production par exemple, le test de Mandel suffit à conclure : les instruments statistiquement semblables peuvent être déclarés conformes, les autres rebutés ou dégradés.

Si la C2I participe d'une surveillance d'étalons, la reproductibilité des instruments ayant passé les tests semble être une donnée importante, à comparer avec leur incertitude d'utilisation. En effet, si la reproductibilité est supérieure à l'incertitude, on peut craindre que la C2I ne soit pas apte à vérifier les qualités attendues des étalons.

Note: afin d'en faciliter la compréhension, le test présenté porte sur un seul niveau $(2 \mathrm{ml})$. En toute rigueur, il devrait porter sur 2 niveaux pour détecter la part variable d'une éventuelle dérive.

\section{Conclusion}

Il est convenu que si la sélection a été correctement réalisée, les instruments écartés devraient en principe faire l'objet d'un étalonnage, voire d'une maintenance.

Les instruments restant à l'issue des tests n'ayant eu, d'une part, aucune raison de dériver de la même manière (hypothèse de départ) et ayant été, d'autre part, étalonnés dans le passé, ne se sont pas «éparpillés 》 dans le temps $\mathrm{du}$ fait qu'ils ne sont pas statistiquement différents (résultat des tests). Leur raccordement est donc encore assuré au moment de la C2I.

En outre, si nous pouvons admettre que les instruments ayant passé les tests sont toujours raccordés, il devient possible, comme nous l'avons vu au paragraphe $\S 6$, de déterminer la variance liée à ce type d'instruments, variance qui peut alors être utilisée dans un calcul d'incertitude d'un processus qui met en œuvre un tel type de moyen. Cette variance, déterminée expérimentalement, semble plus pertinente qu'une évaluation de type $\mathrm{B}$ souvent calculée à partir des résultats d'étalonnage (Erreur maximale constatée) ou de vérification (Erreur Maximale Tolérée). Par ailleurs, il semblerait qu'on puisse régler en partie la question récurrente des « limites d'acceptation (E.M.T)». En effet, une C2I permet finalement de conclure quant à l'homogénéité d'une famille d'instruments, ce qui revient à dire que tous les instruments sont statistiquement identiques entre eux, donc «conformes». Il serait en effet curieux qu'une entreprise ne se soit pas rendu compte que les moyens de mesure qu'elle utilise au quotidien ne sont pas adaptés à son besoin. 